\title{
Correlation between Tonsil Shadow Area on Skull Lateral View and Tonsil Volume after Tonsillectomy
}

\author{
Ho Chan Kim, MD, PhD ${ }^{1}$, Jin Taek Park, MD², Yoo-Sam Chung, MD, PhD² \\ 'Department of Otorhinolaryngology-Head and Neck Surgery, Eulji Medical Center, Eulji University School of Medicine, Seoul, Korea \\ ${ }^{2}$ Department of Otolaryngology, Asan Medical Center, University of Ulsan College of Medicine, Seoul, Korea
}

Received: May 14, 2019

Revised: June 1, 2019

Accepted: June 11, 2019

Correspondence

Yoo-Sam Chung, MD, PhD

Department of Otolaryngology,

Asan Medical Center,

University of Ulsan College of Medicine,

88 Olympic-ro 43-gil, Songpa-gu,

Seoul 05505, Korea

Tel +82-2-3010-3710

Fax +82-2-489-2773

E-mail yschung@amc.seoul.kr

ORCID

Ho Chan Kim

https://orcid.org/0000-0002-3821-5416

Jin Taek Park

https://orcid.org/0000-0003-2013-2062

Yoo-Sam Chung

https://orcid.org/0000-0002-8866-5415
Background and Objective To begin with, an adenotonsillectomy is one of the most common operations in children who have been diagnosed with a condition of sleep-disordered breathing. Upon the preoperative evaluation of tonsil and adenoid size, it is important to predict the probable postoperative outcome. In this study, we investigated the correlation between the subjective tonsil grade, tonsil shadow area on skull lateral view radiographs, and tonsil volume after tonsillectomy.

Methods We retrospectively reviewed the medical records of 234 patients who underwent an adenotonsillectomy in the tertiary referral hospital from January to June 2014. We demarcated the tonsil shadow area on the skull lateral view. When visible, the tonsil shadow area was considered as ellipse. The tonsil area was calculated by measuring the major (2a) and minor (2b) axes of shadow $(\mathrm{S}=\pi \mathrm{ab})$. The tonsil volume was measured at the time of surgery.

Results The tonsil shadow was visible in 180 cases and invisible in 54 cases. The tonsil volume varied from $1.0 \mathrm{~cm}^{3}$ to $9.5 \mathrm{~cm}^{3}$. It is noted that the tonsil volume was significantly greater in the visible group than in the invisible group, based on the lateral radiograph $\left(3.81 \pm 1.21 \mathrm{~cm}^{3}, 3.24 \pm 1.03\right.$ $\mathrm{cm}^{3}$, respectively; $\left.\mathrm{p}=0.002\right)$. In a review of the visible tonsil group $(\mathrm{n}=180)$, there was a positive correlation between the tonsil shadow area and tonsil volume $(r=0.489, p<0.001)$ and between tonsil grade and tonsil volume $(\mathrm{r}=0.239, \mathrm{p}<0.001)$.

Conclusions Tonsillar hypertrophy can be evaluated more accurately when using skull lateral view radiographs as compared with the use of a clinical examination. A tonsil shadow on skull lateral view can be a helpful, non-invasive, and relatively easy tool to predict tonsil volume in many cases.

Sleep Med Res 2019;10(1):8-12

Key Words Tonsillar hypertrophy, Tonsil shadow, Tonsil volume, Adenotonsillectomy.

\section{INTRODUCTION}

Adenotonsillectomy is one of the most common operations for children with obstructive sleep-disordered breathing. In this context, an accurate evaluation of palatine tonsillar hypertrophy and adenoid vegetation is an important predictive factor for the successful treatment of pediatric patients with obstructive sleep apnea (OSA) [1]. For this reason, before performing a tonsillectomy, the conventional tonsil grading system with oropharyngeal examination was the only way to evaluate the degree of tonsillar hypertrophy. However, this subjective tonsil grade has several limitations. In all cases, because it is a subjective measurement, it has low inter-rater reliability and does not include an evaluation of the lower tonsillar poles. The evaluation is only based on a comparison of the tonsillar extension to the anterior pillars, making it difficult to assess the whole tonsil dimensions and volume [2]. The Wang et al. [3] study reported that subjective tonsil size in sleep-disordered breathing children may not reflect the objective tonsil volume in those cases.

In contrast, assessment of the tonsil shadow area is an objective measurement that enables 
the evaluation of the complete tonsil, even in the case of the lower tonsillar poles. It is noted that the skull lateral view radiograph is a useful tool to evaluate adenoid hypertrophy or nasopharyngeal narrowing; however, tonsil shadow also can be observed in the skull lateral view. In addition, this technique can be easily performed on pediatric patients in an out-patient clinic and it is relatively inexpensive [4-7]. In other words, the tonsil shadow is visible in the majority of skull lateral view radiographs of pediatric patients with adenotonsillar hypertrophy (Fig. 1). In this relation, it is noted that in recent years, advances in imaging technology have made it possible to configure image quality by changing the shadow with the picture archiving and communication system tools. In this study, we investigated the correlation between the initial examination of the tonsil, area of tonsil on the skull lateral view, and the tonsil volume after a surgically performed tonsillectomy.

\section{METHODS}

In this case, we retrospectively reviewed medical records of 234 pediatric patients who were diagnosed with tonsillar hypertrophy and underwent adenotonsillectomy in tertiary hospital between January 2014 and June 2014. The Institution's Human Ethics Committee approved this study (application number 20180623). Furthermore, we excluded patients with a previous history of adenotonsillectomy, asymmetric tonsil size, peritonsillar abscess, craniofacial deformity, or Down's syndrome. Additionally, the patients with sleep-disordered breathing, except snoring and sleep apnea, were also excluded from this study. In this case, preoperatively, all of the patients were assigned a tonsil grade using an oropharyngeal exam as follows: grade I, tonsils hidden within the tonsil pillars; grade II, tonsils extending to the pillars; grade III, tonsils extended three-fourths of the way to the midline; grade IV, kissing tonsils.
In this case, a skull lateral view radiograph was obtained for all the subjects preoperatively and the radiograph results were evaluated by two independent otolaryngologists. First, we assessed the visibility of the palatine tonsil shadow on the skull lateral view (Fig. 1). In cases with a visible tonsil shadow, we measured the length of the major axis (2a) and minor axis (2b), and calculated the tonsil shadow area by substituting the formula for an ellipse $(S=\pi \mathrm{ab})$ considering that, generally, palatine tonsils are ellipsoid (Fig. 2). Generally speaking, if there were both tonsil shadows in one radiograph, we choose more delineated one. In the meantime, this method calculating tonsil shadow area on skull lateral view was first proposed by us as far as we know.

Under general anesthesia, each patient underwent the procedure of a bilateral tonsillectomy: after a local anesthetic injection (1\% lidocaine with diluted epinephrine at a ratio of $1: 200,000)$ at both anterior tonsil pillars, the dissection of the tonsils was performed using a monopolar electrosurgical device by one senior surgeon. Therefore, after obtaining the tonsil specimen, we measured the volume of the palatine tonsils with the following method: we measured the increase in saline volume after placing the tonsil into a $20 \mathrm{~cm}^{3}$ syringe tube filled with $10 \mathrm{~cm}^{3}$ saline.

\section{Statistical Analysis}

In this case, the data were analyzed with the SPSS statistical application (SPSS for Windows, release 17.0, SPSS Inc., Chicago, IL, USA), and the figures were plotted with SPSS and Microsoft Office Excel (Microsoft Excel for Windows, release 11.0, 2003, Microsoft Corporation, Redmond, WA, USA). Additionally, an analysis to compare the tonsil volume based on the tonsil shadow and that of the specimen using the independent sample t-test was performed. Next, an interrater reliability for the tonsil shadow area was calculated with the use of a paired t-test. With Spearman's correlation analysis, we obtained the correlation
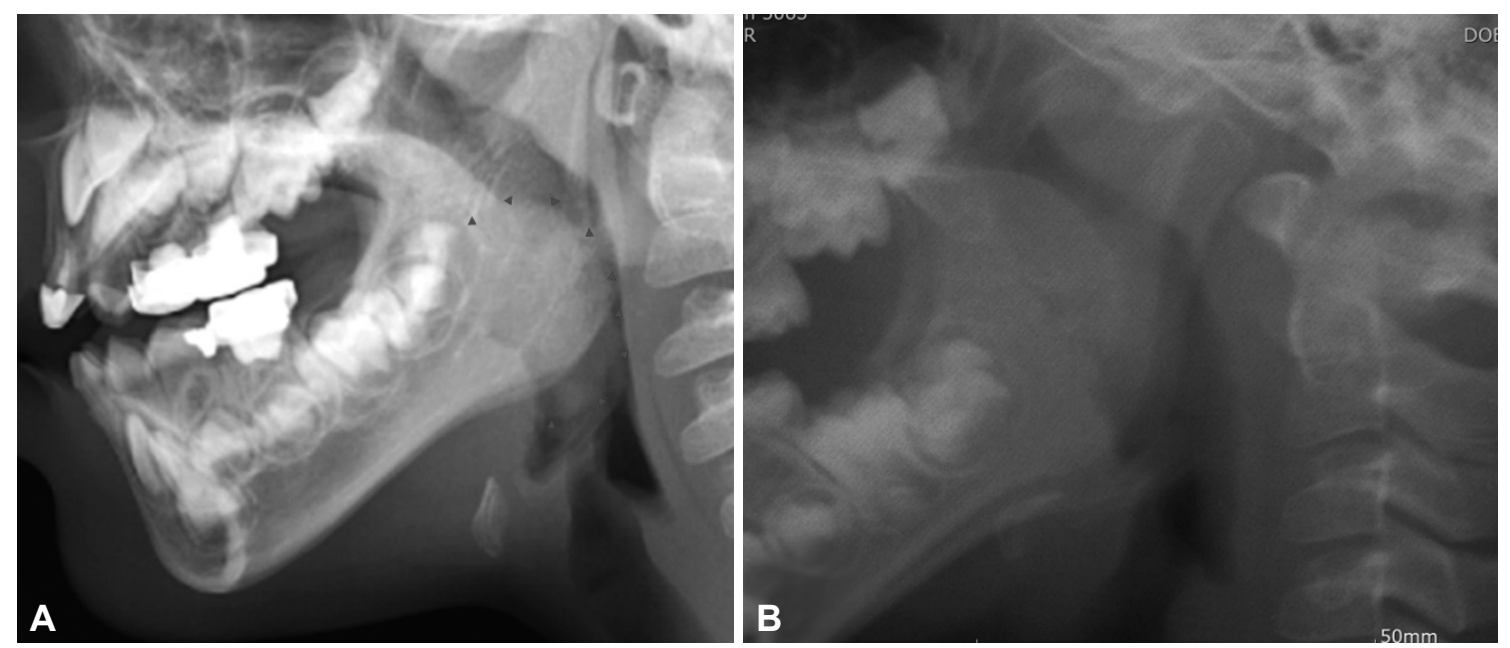

Fig. 1. Tonsil shadow in a skull lateral view radiograph. A: Tonsil shadow is well demarcated (arrowheads) B: Tonsil shadow is invisible. 
coefficients between the Friedman tonsil grade, tonsil shadow area, and tonsil volume. In this case, the p value under 0.05 was considered statistically significant.

\section{RESULTS}

\section{Clinical Characteristics}

In this study, we reviewed the electronic medical records of 234 pediatric patients, retrospectively. The average age at the time of surgery was 5.94 years and $136(58.1 \%)$ patients were male. The number of patients were noted as aged under 5

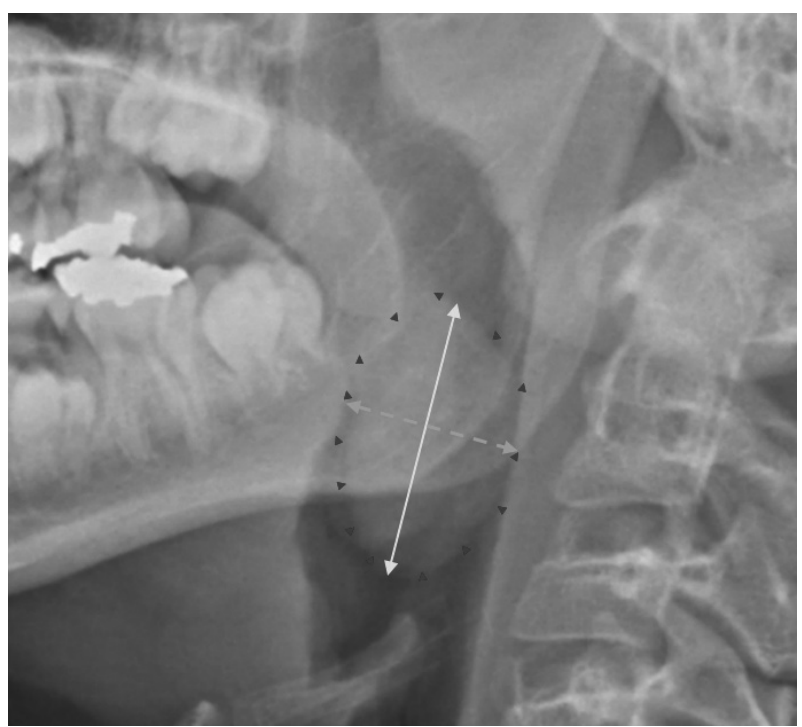

Fig. 2. Calculating tonsil shadow area on the skull lateral view. After demarcating the tonsil shadow margin (arrowheads), length of the major axis (solid line, 2a) and minor axis (broken line, 2b) can be obtained. Tonsil shadow area is $\pi \mathrm{ab}$. years, from 5 to 9 years, and over 10 years, was 70, 140, and 24, respectively. On the oropharyngeal exam, the number of patients for each tonsil grade were as follows: grade I, 5 cases $(2.1 \%)$; grade II, 21 cases (9.0\%); grade III, 126 cases (53.8\%); and grade IV, 82 cases (35.0\%). It is noted that on the skull lateral view, the tonsil shadow was observed in 180 cases (76.9\%). In the visible tonsil shadow group, tonsil grades I, II, III, and IV were assigned in 3 (1.7\%), 11 (6.1\%), 95 (52.8\%), and 71 (39.4\%) cases, respectively (Table 1). Likewise, the determination of the mean tonsil volume was $3.68 \pm 1.19 \mathrm{~cm}^{3}$ and the mean area of tonsil shadow was $3.14 \pm 0.83 \mathrm{~cm}^{2}$. Thus, no significant differences was observed between the scorers (two first authors) for tonsil shadow area $(\mathrm{p}=0.12)$.

\section{Correlation between Tonsil Volume and the Tonsil Grade and Tonsil Shadow Area}

First it is noted that the tonsil volume was significantly larger in the visible tonsil shadow group than in the invisible tonsil shadow group $\left(3.81 \pm 1.21 \mathrm{~cm}^{3}, 3.24 \pm 1.03 \mathrm{~cm}^{3}\right.$, respectively; $\mathrm{p}=0.002)$ (Table 2). Next, in the visible tonsil group $(\mathrm{n}=180)$, there was a statistically significant correlation between the tonsil shadow in the skull lateral view and tonsil volume $(r=0.489$, $\mathrm{p}<0.001$ ) (Fig. 3). Finally, there was also a significant correlation between the tonsil grade and tonsil volume $(\mathrm{r}=0.239, \mathrm{p}<$ 0.001) (Table 3).

\section{DISCUSSION}

The prevailing discipline is that the skull lateral view radiograph is a useful tool to evaluate upper airway obstruction, like adenoid vegetation, however, there are few studies about palatine tonsillar hypertrophy using this radiographic image [4,6,8-11].

Table 1. General characteristics of enrolled patients

\begin{tabular}{|c|c|c|c|c|c|}
\hline \multirow{2}{*}{ Characteristics } & \multirow{2}{*}{$\mathrm{n}=234$} & \multirow{2}{*}{$(\%)$} & \multirow{2}{*}{ Tonsil volume (SD) $\left(\mathrm{cm}^{3}\right)$} & \multicolumn{2}{|c|}{ Tonsil shadow on skull lateral view } \\
\hline & & & & Visible (\%) $\mathrm{n}=180$ & Area $(\mathrm{SD})\left(\mathrm{cm}^{2}\right)$ \\
\hline \multicolumn{6}{|l|}{ Sex } \\
\hline Male & 136 & 58.1 & & & \\
\hline Female & 98 & 41.9 & & & \\
\hline \multicolumn{6}{|l|}{ Age (years) } \\
\hline$<5$ & 70 & 29.9 & & & \\
\hline $5-9$ & 140 & 59.8 & & & \\
\hline$\geq 10$ & 24 & 10.3 & & & \\
\hline \multicolumn{6}{|l|}{ Tonsil grade } \\
\hline I & 5 & 2.1 & $2.3(0.58)$ & $3(1.7)$ & $2.3(0.97)$ \\
\hline II & 21 & 9.0 & $2.9(1.31)$ & $11(6.1)$ & $2.3(0.78)$ \\
\hline III & 126 & 53.8 & $3.76(1.06)$ & $95(52.8)$ & $3.0(0.60)$ \\
\hline IV & 82 & 35.0 & $4.07(1.30)$ & $71(39.4)$ & $3.5(0.94)$ \\
\hline
\end{tabular}

SD: standard deviation. 
The researchers in Pirilä-Parkkinen et al. [12] performed a comparative analyses of the nasopharyngeal and oropharyngeal airway in lateral cephalograms and magnetic resonance imaging (MRI). They reported that most of the cephalometric variables were not significantly related to tonsil grade, except the soft tissue area on the cephalograms. However, in that case they did not identify the exact tonsil portion measured in the cephalograms and only compared MRI results to the radiographs, and not to the actual tonsil volumes.

The Li et al. researchers studied the correlation between the tonsillar-pharyngeal (T-P) ratio and severity of OSA in 35 lateral cephalograms, using the method suggested by Shintani et al. $[13,14]$. They reported that the OSA parameters [apnea-hypopnea index (AHI) and oxygen desaturation index] are correlated positively with the T-P ratio $(r=0.798, p<0.0001$ and $r=0.505$, $p=0.002$, respectively), and emphasized the clinical usefulness of the radiographic T-P ratio. The researchers delineated the tonsil shadow on radiographic imaging, however, they only measured the horizontal length of tonsil shadow, creating only a onedimensional parameter of the tonsil in that case.

To our knowledge, the current study is the first to use the actual volumes of tonsils to compare with the preoperative tonsil shadow area on skull lateral view radiographs in pediatric patients. It is shown that the tonsil shadow area in the skull lateral view had a statistically meaningful correlation with the actual tonsil volume. The conventional tonsil grade also had a positive correlation with tonsil volume, however, the correlation of the tonsil shadow area was shown to have been stronger. The tonsil grade result is a one-dimensional parameter because it only reflects the relative extension of the tonsil to the anterior pillars. In contrast, the tonsil shadow area is a two-dimensional parameter, whereby it can better reflect tonsillar hypertrophy, than with the use of just the tonsil grading system.

That being noted, the upper airway obstruction in OSA patients is caused by static elements and dynamic elements. The static elements are consisted with bony framework and soft tissue volume. In many cases, the dynamic elements are the muscle tone differences during awakening and sleep. In other

Table 2. Tonsil volume differences between visible tonsil shadow group and invisible tonsil shadow group

\begin{tabular}{crcc}
\hline Tonsil shadow & \multicolumn{1}{c}{$\mathrm{n}$} & Tonsil volume $\left(\mathrm{cm}^{3}\right)(\mathrm{SD})$ & $\mathrm{p}$-value \\
\hline Visible & 180 & $3.81(1.21)$ & 0.002 \\
Invisible & 54 & $3.24(1.03)$ & \\
\hline
\end{tabular}

SD: standard deviation. words, the pediatric tonsillectomy is an operation characteristic of widening the upper airway by reducing one of the static elements, namely the soft tissue volume. With preoperative estimating of tonsil volume, a surgeon can predict how effective will be the widening transection area of the upper airway. Until now, a review of the subjective tonsil grade is most commonly used as an evaluation tool by many physicians. However, low pole of tonsils are obscured by tongue and deep buried portion of tonsil, and therefore cannot be evaluated with tonsil grades. It is difficult to reflect the difference in detail, because the tonsil grade has only 4 distinguishable grades.

It is noted that in 45 patients (23.1\%), a tonsil shadow was invisible upon review. And in those cases, a real tonsil volume was noted as being larger in the visible tonsil group than in the invisible tonsil group. Proportionally, a small tonsil volume may have narrow tonsillar width, inadequate to make distinct tonsil shadow on skull lateral view. On the basis of this result, pediatric OSA patients with invisible tonsil shadow may have a small tonsil volume. In addition, it is shown in this case as $0.01 \mathrm{mSv}$ radiation exposure per one skull lateral view, and it only noted $0.79 \%$ of natural background radiation per year (1.26 mSv) [15]. The procurement of a skull lateral view is a relatively easy and safe modality to the evaluation of the tonsil volume in pediatric OSA patients, preoperatively.

There is debate about the correlation between subjective tonsil size, tonsil volume and OSA severity in previous researches. The Tang et al. [16] study reported that subjective tonsil grade did not correlate with OSA severity in children,

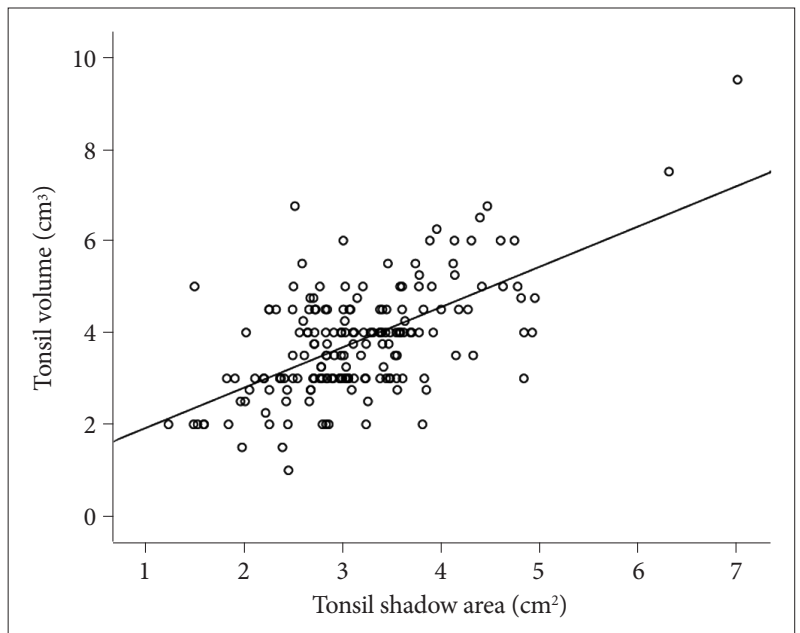

Fig. 3. Scatter plot of the correlation analysis between the area of tonsil shadowing and tonsil volume $(r=0.489, p<0.001)$.

Table 3. Comparison of correlation analysis between tonsillar hypertrophy, tonsil shadowing area, and tonsil volume $(n=180)$

\begin{tabular}{lcr}
\hline & Spearman correlation coefficient & p-value \\
\hline PTH grade vs. tonsil volume & 0.239 & 0.001 \\
Area of tonsil shadow vs. tonsil volume & 0.489 & $<0.001$ \\
\hline
\end{tabular}

PTH: palatine tonsillar hypertrophy. 
and the systematic review of the Nolan et al. [17] study also concluded that the correlation between the identified subjective tonsil grade and OSA severity is considered to be statistically weak. On the other hand, Cahali et al. [18] reported that the objective tonsil volume had a positive correlation with AHI and the Epworth Sleepiness Scale in adult. In this relation, the Jara et al. [19] reported that subjective tonsil grade is more strongly correlated with OSA severity than real tonsil volume in adults. By the same token, Verse et al. [20] reported that adult OSA patients with substantial tonsillar hypertrophy showed significant improvement even after receiving of the tonsillectomy procedure alone. Further studies regarding real tonsil volume, tonsil size and OSA severity in pediatric patients would be needed.

As noted, there are several limitations to this study. First, this was a retrospective study and we did not evaluate the improvements of obstructive sleep apnea severity as associated with preoperative and postoperative polysomnography. In this study, the issue of adenoid vegetation, one of the main influencing factors for pediatric sleep apnea, was also not evaluated. Second, we did not control for the incidence or condition of soft tissue thickness, such as body mass index or neck circumference or overlapping bony structures, like mandible, as noted on the tonsil shadow area. Incidentally, the presence of thick soft tissue and bony structures can make the tonsil shadow vague, which makes it difficult to delineate the tonsil shadow on the skull lateral view. Lastly, there were 54 patients with invisible tonsil shadows, and we could not evaluate the correlation between tonsil volume and tonsil shadow in these patients.

In conclusion, the image of a tonsil shadow area as noted on the skull lateral view radiographs tends to better reflect the actual tonsil volume than the subjective tonsil grading system; therefore, the tonsil shadow area can be helpful for predicting real tonsil volume preoperatively for pediatric patients with diagnosed sleep-disordered breathing. Further studies involving tonsil shadows, other anatomic factors of sleep apnea, and severity of sleep apnea would be needed to develop other suitable options for patients with this condition.

\section{Conflicts of Interest}

The authors have no financial conflicts of interest.

\section{Authors' Contribution}

Conceptualization: Chung YS. Data collection: Kim HC, Park JT. Formal analysis: Kim HC, Park JT. Methodology: Chung YS, Kim HC. Project administration: Chung YS, Park JT. Supervision: Chung YS. Writingoriginal draft: Kim HC, Park JT. Writing-review \& editing: Chung YS, Kim HC.

\section{REFERENCES}

1. Mitchell RB. Adenotonsillectomy for obstructive sleep apnea in children: outcome evaluated by pre- and postoperative polysomnography. Laryngoscope 2007;117:1844-54.

2. Patel NA, Carlin K, Bernstein JM. Pediatric airway study: Endoscopic grading system for quantifying tonsillar size in comparison to standard adenotonsillar grading systems. Am J Otolaryngol 2018;39:56-64.

3. Wang JH, Chung YS, Jang YJ, Lee BJ. Palatine tonsil size and its correlation with subjective tonsil size in patients with sleep-disordered breathing. Otolaryngol Head Neck Surg 2009;141:716-21.

4. Hwang SH, Guilleminault C, Park CS, Kim TW, Hong SC. Usefulness of adenotonsillar size for prediction of severity of obstructive sleep apnea and flow limitation. Otolaryngol Head Neck Surg 2013;149:326-34.

5. Kim DK, Rhee CS, Yun PY, Kim JW. Adenotonsillar hypertrophy as a risk factor of dentofacial abnormality in Korean children. Eur Arch Otorhinolaryngol 2015;272:3311-6.

6. McNamara JA Jr. A method of cephalometric evaluation. Am J Orthod Dentofacial Orthop 1984;86:449-69.

7. Diouf JS, Ngom PI, Sonko O, Diop-Bâ K, Badiane A, Diagne F. Influence of tonsillar grade on the dental arch measurements. Am J Orthod Dentofacial Orthop 2015;147:214-20.

8. Major MP, Flores-Mir C, Major PW. Assessment of lateral cephalometric diagnosis of adenoid hypertrophy and posterior upper airway obstruction: a systematic review. Am J Orthod Dentofacial Orthop 2006; 130:700-8.

9. Zicari AM, Duse M, Occasi F, Luzzi V, Ortolani E, Bardanzellu F, et al. Cephalometric pattern and nasal patency in children with primary snoring: the evidence of a direct correlation. PLoS One 2014;9:e111675.

10. Ozdemir H, Altin R, Söğüt A, Cinar F, Mahmutyazicioğlu K, Kart L, et al. Craniofacial differences according to AHI scores of children with obstructive sleep apnoea syndrome: cephalometric study in 39 patients. Pediatr Radiol 2004;34:393-9.

11. Sousa JB, Anselmo-Lima WT, Valera FC, Gallego AJ, Matsumoto MA. Cephalometric assessment of the mandibular growth pattern in mouthbreathing children. Int J Pediatr Otorhinolaryngol 2005;69:311-7.

12. Pirilä-Parkkinen K, Löppönen H, Nieminen P, Tolonen U, Pääkkö E, Pirttiniemi P. Validity of upper airway assessment in children: a clinical, cephalometric, and MRI study. Angle Orthod 2011;81:433-9.

13. Shintani T, Asakura K, Kataura A. Adenotonsillar hypertrophy and skeletal morphology of children with obstructive sleep apnea syndrome. Acta Otolaryngol Suppl 1996;523:222-4.

14. Li AM, Wong E, Kew J, Hui S, Fok TF. Use of tonsil size in the evaluation of obstructive sleep apnoea. Arch Dis Child 2002;87:156-9.

15. Wall BF, Hart D. Revised radiation doses for typical X-ray examinations. Report on a recent review of doses to patients from medical Xray examinations in the UK by NRPB. National Radiological Protection Board. Br J Radiol 1997;70:437-9.

16. Tang A, Benke JR, Cohen AP, Ishman SL. Influence of tonsillar size on OSA improvement in children undergoing adenotonsillectomy. Otolaryngol Head Neck Surg 2015;153:281-5.

17. Nolan J, Brietzke SE. Systematic review of pediatric tonsil size and polysomnogram-measured obstructive sleep apnea severity. Otolaryngol Head Neck Surg 2011;144:844-50.

18. Cahali MB, Soares CF, Dantas DA, Formigoni GG. Tonsil volume, tonsil grade and obstructive sleep apnea: is there any meaningful correlation? Clinics (Sao Paulo) 2011;66:1347-52.

19. Jara SM, Weaver EM. Association of palatine tonsil size and obstructive sleep apnea in adults. Laryngoscope 2018;128:1002-6.

20. Verse T, Kroker BA, Pirsig W, Brosch S. Tonsillectomy as a treatment of obstructive sleep apnea in adults with tonsillar hypertrophy. Laryngoscope 2000;110:1556-9. 